\title{
Dosimetría por imagen en radioembolización hepática con Y90: impacto de la resolución espacial del PET**
}

\section{Image dosimetry for Y90 hepatic radioembolization: impact of PET spatial resolution}

\author{
Nuria Carrasco Vela ${ }^{1}$, Natalia Tejedor Aguilar², Vicente Giménez Gómez ${ }^{3}$, Irene Torres Espallardo² \\ 1 Hospital Universitario Dr. Peset, Avenida de Gaspar Aguilar, 90, 46017, Valencia. \\ 2 Hospital Universitario y Politécnico La Fe, Avenida de Fernando Abril Martorell, 106, 46026, Valencia. \\ 3 Universidad de Valencia - Instituto de Física Corpuscular (IFIC). Calle del Catedrático José Beltrán Martinez, 2, 46980 \\ Paterna, Valencia.
}

Fecha de Recepción: 29/08/2021 - Fecha de Aceptación: 13/09/2021

La imagen PET es crucial en la dosimetría de tratamientos de radioembolización hepática con Y90. Sin embargo, dada la limitada resolución espacial de estos escáneres, la distribución real de actividad se difumina. Suele argumentarse que este difuminado es mayor que el debido a la convolución con un kernel de dosis (algoritmo VSV) de tal modo que el método de deposición local (LDM) proporciona resultados suficientemente precisos. En este trabajo hemos comparado los resultados dosimétricos de LDM, VSV y Monte Carlo en imágenes PET generadas matemáticamente a las cuales se les ha aplicado difuminados que simulan la resolución espacial del PET.

Palabras clave: Radioembolización hepática, dosimetría por imagen, resolución espacial PET.

PET image plays a key role in quantitative image-based dosimetry for hepatic Y90 radioembolization. Nevertheless, given the limited scanner spatial resolution, the real activity distribution is blurred. It is usually argued that the blurring introduced by the PET is larger than the one due to the convolution kernel (VSV method) so that Local Deposition Method (LDM) provides sufficiently precise results. In the present work we have compared dosimetric results from LDM, VSV and Monte Carlo on synthetic PET images which have been blurred in order to simulate PET spatial resolution.

Key words: Hepatic radioembolization, image-dosimetry, PET spatial resolution.

\section{Introducción}

La radioembolización (RE) con microesferas de Itrio-90 (Y90) es una prometedora técnica dirigida a la terapia selectiva de cánceres de hígado quimioresistentes o no resecables. Desde hace más de 20 años la RE con Y90 ha usado dos tipos de microesferas: microesferas de vidrio (TheraSphere, Boston Scientific, Marlborough, MA, USA) o microesferas de resina (SIR-Sphere, Sirtex Medical, Australia). El tratamiento consiste en la administración de Y90 a través de la vasculatura de la arteria hepática. Debido a la diferenciada vascularización del hígado sano y el tumor, las microesferas se acumulan preferentemente en el tejido tumoral dando lugar a una irradiación local del tumor liberando el tejido hepático sano.

El Y90 es un emisor beta puro de alta energía (energia máxima 2.3 MeV y energía media $0.923 \mathrm{MeV}$ ), vida media de 2.67 días y un poder de penetración en tejido de $11 \mathrm{~mm}$. Además, la verificación cuantitativa por imagen es posible tanto en el SPECT, a partir de la radiación de frenado inducida, como en el PET, explotando la

\footnotetext{
*Correspondencia: carrasco_nur@gva.es

**Trabajo presentado en el $3^{\text {er }}$ Congreso Europeo de Física Médica (ECMP) (16-19 Junio, Turín, Italia) y becado por la SEFM para la asistencia al mismo.

https://doi.org/10.37004/sefm/2021.22.2.004
} 
pequeña fracción de desintegración a positrones (32 ppm).

Recientemente se han publicado los resultados del ensayo clínico DOSISPHERE-01 ${ }^{1}$ que compara, en pacientes con hepatocarcinoma, la dosimetría estándar, basada en la administración de 120 Gy al lóbulo perfundido, frente a la dosimetría personalizada que tiene en cuenta la diferente captación del tejido tumoral y sano dentro del volumen perfundido. La proporción de pacientes con respuesta completa o parcial es significativamente mayor en el grupo que se abordó con dosimetría personalizada, sin incremento de la toxicidad. Estos alentadores resultados han motivado a la comunidad científica a impulsar la calidad y precisión en la dosimetría de los tratamientos de RE con Y90.

Actualmente, los métodos para dosimetría personalizada basada en imagen para la RE con Y90 están basados en el modelo multicompartimental ${ }^{2}$ donde el único parámetro dosimétrico evaluado en cada compartimento (tumor o no tumor) es el valor medio de la dosis en dicho compartimento. La principal ventaja de este modelo es su simplicidad. Ahora bien, el modelo de partición es limitado ya que ignora la distribución no uniforme de actividad en cada volumen. Este inconveniente puede solventarse utilizando dosimetrías voxelizadas. Entre los métodos de dosimetría 3D destacan el método de deposición local de dosis (LDM), el de convolución, también conocido como Voxel-S-Value (VSV) y el método de Monte Carlo (MC).

El método de convolución o VSV ${ }^{3}$ considera que la dosis absorbida en cada vóxel es debida a la actividad presente en dicho vóxel más la contribución debida a los vóxeles vecinos, lo que se traduce en que la distribución de dosis se obtiene convolucionando la matriz tridimensional de distribución de actividad obtenida a partir de la imagen con la matriz de convolución de dosis. Estas matrices de convolución han sido calculadas previamente mediante técnicas de MC para distintos tamaños de vóxeles. Por su parte, el método LDM ${ }^{4}$ asume que cada partícula deposita la energía en el vóxel en el cual se ha generado. Con esta simplificación, en el método LDM la dosis en cada vóxel se calcula simplemente multiplicando la distribución de actividad por un factor. Este factor no es más que la dosis absorbida en cada vóxel por cada desintegración, estimada a partir de la energía media de los electrones emitidos, por unidad de masa.

A día de hoy está aceptado que el método de MC es el más exacto a la hora de modelizar el transporte de la radiación. Sin embargo, la necesidad de considerar un número suficiente de eventos en la simulación implica en general un excesivo coste computacional y es por ello que las simulaciones de MC están muy restringidas en la rutina clínica.

La imagen PET juega un papel crucial para la dosimetría cuantitativa post-tratamiento en la radioemboli- zación hepática con Y90. A pesar de la baja probabilidad de desintegración a positrones (32ppm) y la alta fracción de coincidencias aleatorias (superior al 901\%), diversos estudios han confirmado el potencial de obtener información cuantitativa de la imagen PET en los tratamientos de radioembolizacion hepática con Y90. En concreto, el estudio QUEST ${ }^{5}$ concluyó que, dentro del rango de actividades usados en la clínica, todos los escáneres PET actuales de GE, Siemens o Philips son capaces de reconstruir y cuantificar la concentración de actividad dentro de una tolerancia de alrededor del $10 \%$.

No obstante, también es cierto que, dada la resolución espacial del PET, la distribución real de actividad se ve difuminada y por tanto la distribución que se utiliza para la dosimetría no corresponde exactamente con la distribución real en el o la paciente.

Suele argumentarse que el difuminado o blurring de la distribución de actividad debido a la resolución espacial del equipo es mayor que el de la convolución con el kernel de dosis de modo que el transporte de energía entre vóxeles vecinos está tenido en cuenta por la propia función de respuesta (PSF, point spread function) del equipo. Por ello, se afirma que LDM aporta resultados suficientemente precisos siendo además más sencillo de implementar.

Como se describirá a continuación, en este trabajo hemos comparado los resultados dosimétricos de los métodos LDM y VSV frente a MC para imágenes PET sintéticas generadas matemáticamente y a las cuales les hemos aplicado difuminados gaussianos de distinta amplitud. El objetivo ha sido estudiar la exactitud de los métodos LDM y VSV en función de la resolución espacial del equipo PET.

\section{Material y métodos}

Usando programas propios en Matlab se generaron imágenes PET con voxelizado $4 \mathrm{~mm}$. Se han considerado las siguientes distribuciones de actividad: tumores de 4,8 y $24 \mathrm{~mm}$ de diámetro y uno o varios tumores de $5 \mathrm{~cm}$ de diámetro con y sin necrosis y con sin fondo de actividad. Estas imágenes corresponderían a distribuciones de actividad ideales, sin el efecto de la PSF del equipo (es decir con $F W H M=0 \mathrm{~mm}$ ). En particular, para los tumores necrosados se ha considerado una corteza de $6 \mathrm{~mm}$ con actividad en la corteza 80 veces superior a la actividad en el interior mientras que para el fondo de actividad se ha considerado un cociente tumor/no tumor (TNT) igual a 2.

A continuación, utilizando de nuevo programas propios en Matlab, se aplicaron kernels gaussianos de $\mathrm{FWHM}=4,7$ y $10 \mathrm{~mm}$ (ver fig. 1) sobre las imágenes previas con el objetivo de simular el efecto de la resolución espacial del PET. 

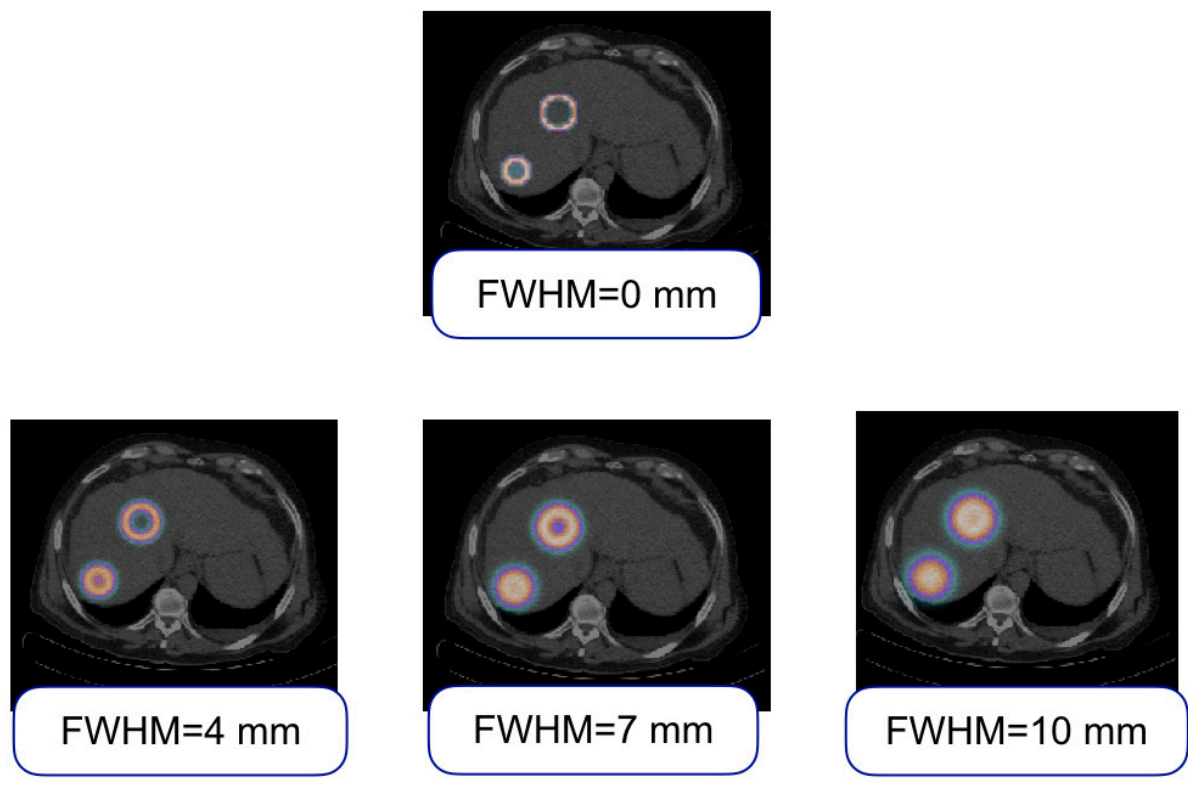

Fig. 1. Ejemplo del proceso seguido para generar imágenes PET sintéticas con difuminado espacial. Partiendo de distribuciones de actividad matemáticamente definidas se aplican kernels gaussianos de 4, 7 y 10 mm de FWHM para simular la resolución espacial del equipo.

Finalmente, se calculó el mapa de dosis con cada uno de los algoritmos tanto para las imágenes difuminadas como para las imágenes sintéticas originales. La dosimetría 3D con LDM y VSV fue realizada con programas propios en Matlab mientras que las simulaciones MC se realizaron con GATE v8.2 considerando CT de pacientes como distribuciones de atenuación. Las imágenes CT consideradas fueron obtenidas con un equipo Philips GEMINI TF PET/CT. Estas simulaciones fueron realizadas utilizando la infraestructura GRID del Instituto de Física Corpuscular de Valencia.

\section{Resultados}

La fig. 2 compara, para distintos casos de imágenes, la dosis en el volumen de interés (VOI) correspondiente al tumor, definido como aquella región de la imagen con actividad no nula, calculada con LDM para cada uno de los difuminados considerados $F W H M=0,4,7$ y $10 \mathrm{~mm}$ frente a la obtenida con una simulación de $\mathrm{MC}$ realizada sobre la imagen con resolución espacial perfecta, es decir, sin ningún tipo de difuminado. Para resoluciones espaciales inferiores

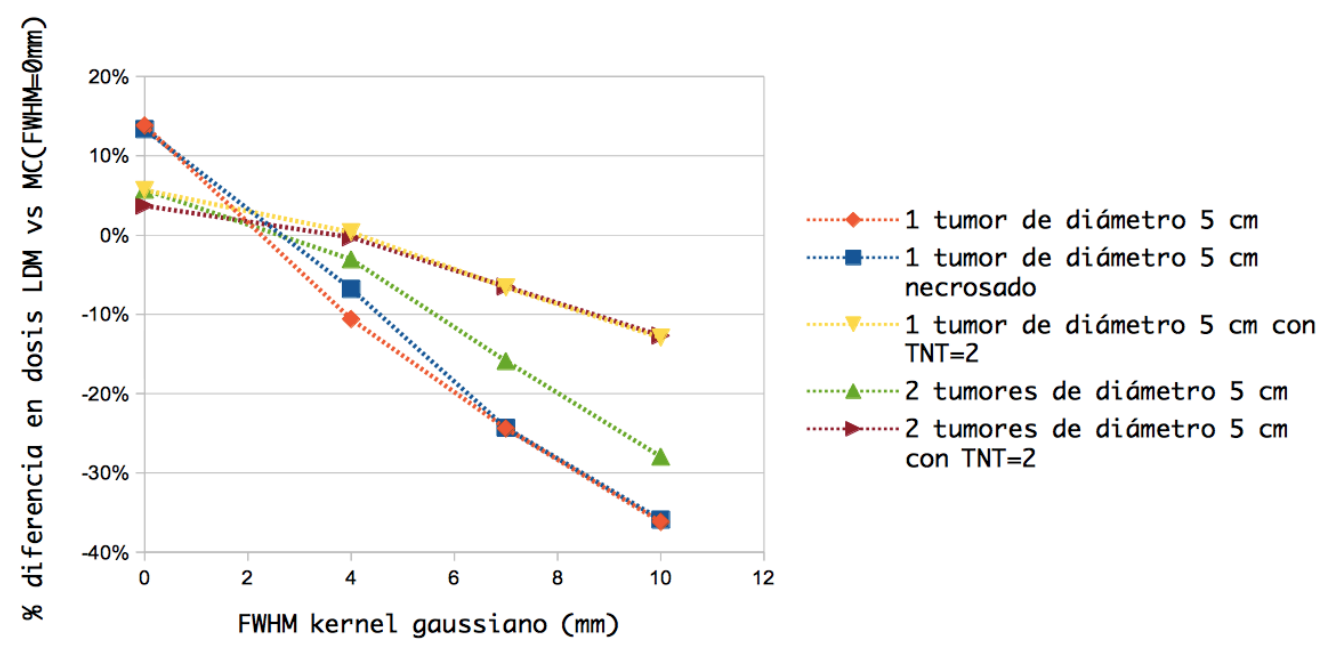

Fig. 2. Diferencia entre la dosis en la VOI del tumor calculada según LDM aplicado a las imágenes con diferentes resoluciones espaciales frente al MC aplicado a la imagen con resolución espacial perfecta $(F W H M=0)$ para diferentes distribuciones de actividad. 

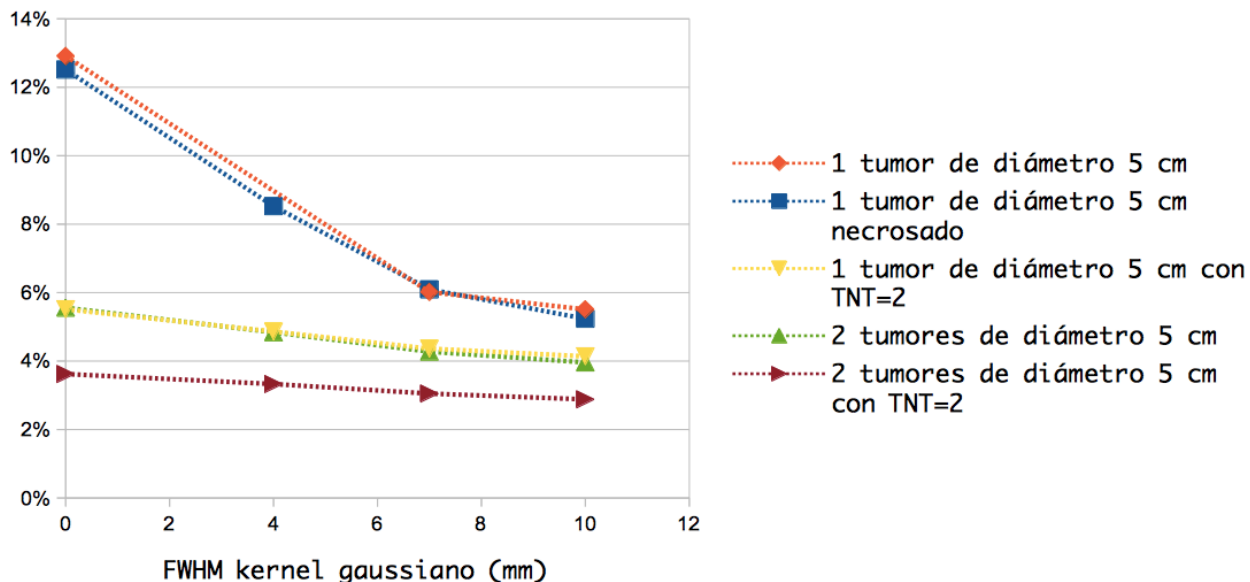

Fig. 3. Diferencia entre la dosis en la VOI del tumor calculada según LDM frente a MC para cada resolución espacial y tipo de distribución de actividad considerada.

a 2-4 mm LDM sobreestima la dosis mientras que para valores de la resolución espacial por encima de 2-4 mm infraestima.

Ahora bien, en la práctica no se dispone de la distribución con una resolución espacial perfecta para utilizarla como gold standard sino que lo más a lo que podemos aspirar es a hacer un MC sobre la imagen PET obtenida con la PSF del equipo. Podemos realizar una gráfica similar a la anterior pero comparando la dosis en la VOI según LDM y MC para cada una de las resoluciones espaciales consideradas (ver fig. 3). En este caso, observamos que al disminuir el difuminado aumenta la diferencia entre ambos métodos pudiendo alcanzar diferencias mayores al $5 \%$ para resoluciones espaciales de $4 \mathrm{~mm}$, especialmente cuando la actividad se encuentra más concentrada.

Como ejemplo, la tabla 1 muestra las diferencias de dosis en la VOI del tumor para el caso de un tumor esférico de diámetro $5 \mathrm{~cm}$ necrosado. En dicha tabla se resume la diferencia relativa de dosis media con cada par de métodos. Las diferencias entre VSV y LDM disminuyen al empeorar la resolución espa-

Tabla 1. Diferencias de dosis entre los distintos métodos en la VOI del tumor, para un tumor esférico necrosado de diámetro $5 \mathrm{~cm}$.

\begin{tabular}{c|c|c|c} 
FWHM(mm) & LDM-MC & VSV-MC & LDM-VSV \\
\hline 0 & $12.5 \%$ & $5.6 \%$ & $6.9 \%$ \\
\hline 4 & $8.5 \%$ & $4.5 \%$ & $4.0 \%$ \\
\hline 7 & $6.1 \%$ & $4.0 \%$ & $2.1 \%$ \\
\hline 10 & $5.2 \%$ & $3.8 \%$ & $1.4 \%$ \\
\hline
\end{tabular}

cial. Lo mismo ocurre con las diferencias entre LDM o VSV y MC siendo siempre VSV más cercano a MC.

Pasamos ahora a comparar las diferencias a nivel vóxel. Se muestra como ejemplo uno de los casos con mayores diferencias: el caso del tumor necrosado. La fig. 4 muestra el mapa de diferencias de dosis y matriz gamma $1 \% / 4 \mathrm{~mm}$ correspondientes a la comparación LDM frente MC para un corte axial de este PET sintético sin difuminado (a) y con FWHM=7 mm (b).

Finalmente, la tabla 2 resume el porcentaje de $\Gamma(1 \%, 4 \mathrm{~mm})<1$ para el caso del tumor esférico necrosado. Puede observarse que, independiente de la anchura del kernel gaussiano aplicado, las diferencias en dosis son menores al $1 \%$ en aproximadamente más del $90 \%$ de los vóxeles.

Tabla 2. Porcentaje $\Gamma(1 \%, 4 \mathrm{~mm})<1$ para el caso de un tumor esferico de $5 \mathrm{~cm}$ de diámetro necrosado. Se han excluido del análisis $\Gamma$ aquellos vóxeles cuya dosis es inferior al $1 \%$ del máximo.

\begin{tabular}{c|c|c|c} 
FWHM(mm) & LDM-MC & VSV-MC & LDM-VSV \\
\hline 0 & $89 \%$ & $97 \%$ & $96 \%$ \\
\hline 4 & $100 \%$ & $100 \%$ & $100 \%$ \\
\hline 7 & $99 \%$ & $99 \%$ & $100 \%$ \\
\hline 10 & $95 \%$ & $95 \%$ & $100 \%$
\end{tabular}

\section{Discusión}

Aunque sería necesario simular más tipos de imágenes PET sintéticas, otros valores de difuminado 

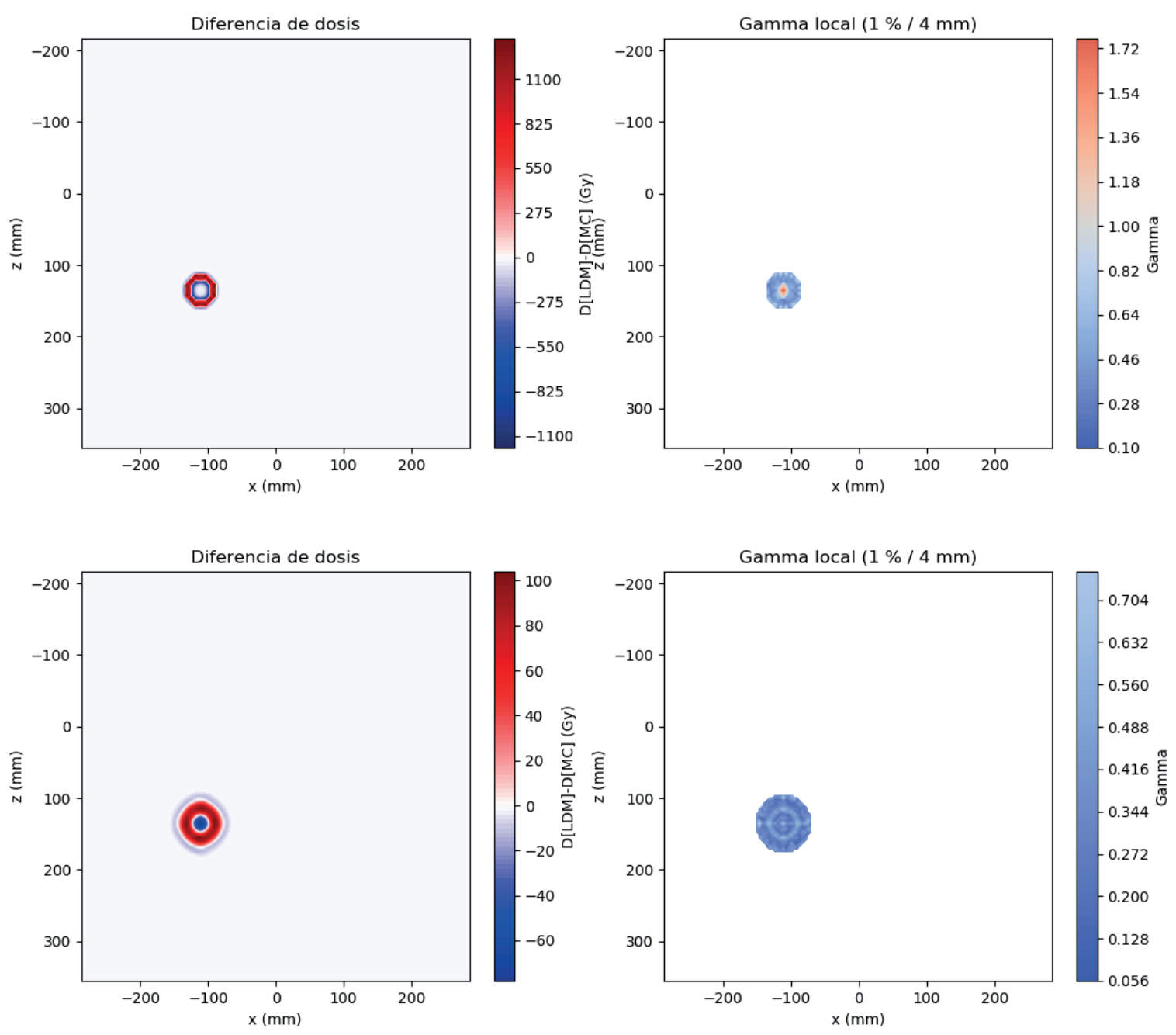

Fig. 4. Diferencias de dosis (en Gy) y matriz gamma 1\%,4 mm de la comparación MC frente a LDM para un corte axial de la imagen PET correspondiente al tumor necrosado de $5 \mathrm{~cm}$ de diámetro sin difuminado (fila superior) y aplicando un kernel gaussiano con $\mathrm{FWHM}=7 \mathrm{~mm}$ (inferior). Se asume una actividad administrada de $2 \mathrm{GBq}$.

gaussiano y voxelizados más pequeños de $4 \mathrm{~mm}$, observamos que en general la resolución espacial del equipo PET conlleva diferencias de dosis a nivel vóxel menores del $1 \%$. A nivel VOI, al aumentar el difuminado, es decir al empeorar la resolución espacial del equipo, disminuye la diferencia entre VSV y LDM y ambos tienden a MC siendo siempre VSV más cercano a MC.

Por otra parte, comparados con la simulación de $\mathrm{MC}$ sobre la imagen PET con FWHM=0mm, lo que podriamos considerar el gold standard, podemos destacar que para resoluciones espaciales por encima de 2-4 mm LDM infraestima la dosis mientras que por debajo de 2-4 mm la sobrestima. Esto es debido a que, cuando la resolución espacial es mayor que 2-4 mm, el difuminado de la distribución de actividad que implica es mayor que el alcance de los electrones incluido en el cálculo de MC.

\section{Conclusiones}

La resolución espacial de los PET actuales está en el rango 7-10 mm 6-8 mientras que con equipos más modernos se espera llegar a $4 \mathrm{~mm}^{9}$ En este rango esperamos dosis a nivel vóxel similares (al 1\%) entre los distintos métodos, pero se debería prestar atención al considerar la dosis media donde al comparar LDM con MC se podría alcanzar, para ciertas distribuciones de actividad, diferencias cercanas al 10\% para resoluciones espaciales de $4 \mathrm{~mm}$ y menores. Sin embargo, cabe destacar que, para resoluciones espaciales del PET en torno a $4 \mathrm{~mm}$, LDM ofrece resultados muy cercanos al gold standard, es decir la simulación de MC sobre la imagen PET sintética con resolución espacial perfecta. 


\section{Referencias}

1. Garin E, Tselikas L, Guiu B, et al. Personalised versus standard dosimetry approach of selective internal radiation therapy in patients with locally advanced hepatocellular carcinoma (DOSISPHERE-01): a randomised, multicentre, open-label phase 2 trial. The Lancet Gastroenterology \& Hepatology 2021 Jan; 6:17\{29. doi: 10.1016/s24681253(20)30290-9. Available from: https://doi.org/10.1016/ s2468-1253(20)30290-9

2. Ho S, Lau WY, Leung TWT, Chan M, Ngar YK, Johnson PJ, and $\mathrm{Li}$ AKC. Partition model for estimating radiation doses from yttrium-90 microspheres in treating hepatic tumours. European Journal of Nuclear Medicine 1996; 23:947\{52. doi: 10.1007/bf01084369. Available from: https://doi. org/10.1007/bf01084369

3. Bolch W, Bouchet L, Robertson J, Wessels B, Siegel J, Howell R, Erdi A, Aydogan B, Costes S, Watson E, Brill A, Charkes N, Fisher D, Hays M, and Thomas S. MIRD pamphlet No. 17: the dosimetry of nonuniform activity distributions\{radionuclide $S$ values at the voxel level. Medical Internal Radiation Dose Committee. Journal of nuclear medicine : ocial publication, Society of Nuclear Medicine 1999; 40:11S\{36S. Available from: http://europepmc.org/abstract/MED/9935083

4. Pasciak AS, Bourgeois AC, and Bradley YC. A Comparison of Techniques for 90Y PET/CT Image-Based Dosimetry Following Radioembolization with Resin Microspheres. Frontiers in Oncology 2014 May; 4. doi: 10.3389/fonc.2014.00121. Available from: https://doi.org/10.3389/fonc.2014. 00121
5. Willowson KP, Tapner M, and Bailey DL. A multicentre comparison of quantitative 90Y PET/CT for dosimetric purposes after radioembolization with resin microspheres. European Journal of Nuclear Medicine and Molecular Imaging 2015 May; 42:1202\{22. doi: 10.1007/s00259-015-3059-9. Available from: https://doi.org/10.1007/s00259-015-3059-9

6. Werner MK, Parker JA, Kolodny GM, English JR, and Palmer MR. Respiratory Gating Enhances Imaging of Pulmonary Nodules and Measurement of Tracer Uptake in FDG PET/CT. American Journal of Roentgenology 2009 Dec; 193:1640\{5. doi: 10.2214/ajr.09.2516. Available from: https://doi. org/10.2214/ajr.09.2516

7. Elmbt $L$ van, Vandenberghe $S$, Walrand $S$, Pauwels $S$, and Jamar F. Comparison of yttrium-90 quantitative imaging by TOF and non-TOF PET in a phantom of liver selective internal radiotherapy. Physics in Medicine and Biology 2011 Oct; 56:6759\{77. doi: 10.1088/0031-9155/56/21/001. Available from: https://doi.org/10.1088/0031-9155/56/21/001

8. Bagni O, D'Arienzo M, Chiaramida P, Chiacchiararelli L, Cannas P, D'Agostini A, Cianni R, Salvatori R, and Scopinaro F. 90Y-PET for the assessment of microsphere biodistribution after selective internal radiotherapy. Nuclear Medicine Communications 2012 Feb; 33:198\{204. doi: 10.1097/mnm. Ob013e32834dfa58. Available from: https://doi.org/10.1097/ mnm.0b013e32834dfa58

9. Delso G, Fürst S, Jakoby B, Ladebeck R, Ganter C, Nekolla SG, Schwaiger M, and Ziegler SI. Performance Measurements of the Siemens mMR Integrated Whole-Body PET/MR Scanner. Journal of Nuclear Medicine 2011 Nov; 52:1914-22. doi: 10.2967/jnumed.111.092726. Available from: https://doi.org/10.2967/jnumed.111.092726 


\section{¿En qué se basa nuestra innovación en iCT? En las necesidades de diagnóstico de más de $\mathbf{6 . 7 0 0}$ millones de personas.}

Del mismo modo que las enfermedades afectan a todos por igual, Brilliance iCT de Philips se adapta a todo tipo de pacientes. Adquiere imágenes nítidas de cualquier parte del cuerpo, desde lactantes de poco peso hasta adultos con

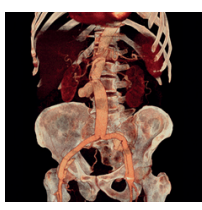

sobrepeso. Los pacientes recibirán el grado de calidad que se merecen en sus exploraciones mediante una dosis baja sin que ello afecte a la calidad de la imagen. Visite nuestra página web en www.philips.com/CT.

*Porque nuestras innovaciones se inspiran en usted.

PHILIPS

sense and simplicity

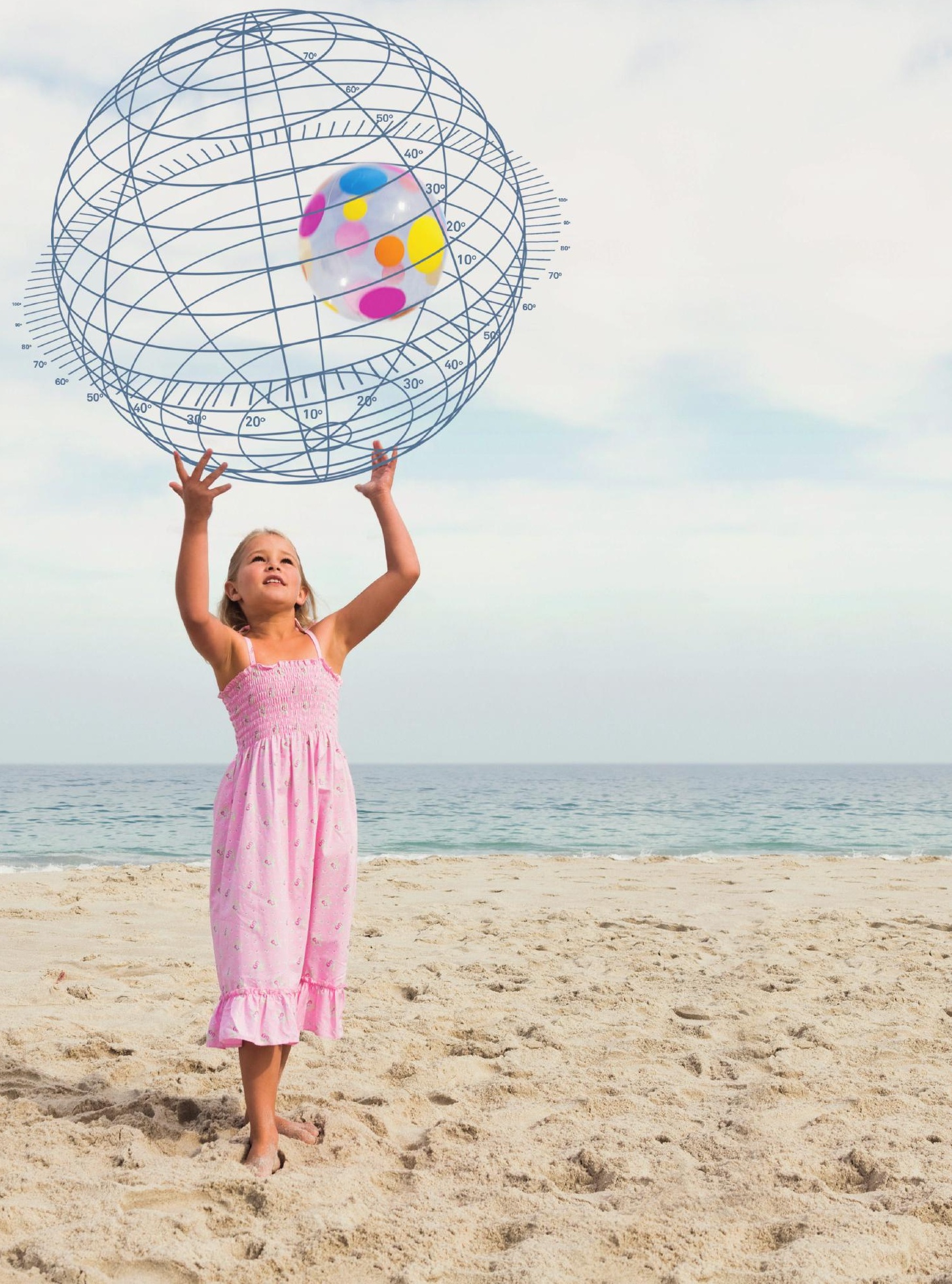

